\title{
Phenotype prediction for mucopolysaccharidosis type I by in silico analysis
}

\author{
Li Ou ${ }^{1 *}$, Michael J. Przybilla ${ }^{2}$ and Chester B. Whitley ${ }^{1,2}$
}

\begin{abstract}
Background: Mucopolysaccharidosis type I (MPS I) is an autosomal recessive disease due to deficiency of a-L-iduronidase (IDUA), a lysosomal enzyme that degrades glycosaminoglycans (GAG) heparan and dermatan sulfate. To achieve optimal clinical outcomes, early and proper treatment is essential, which requires early diagnosis and phenotype severity prediction.

Results: To establish a genotype/phenotype correlation of MPS I disease, a combination of bioinformatics tools including SIFT, PolyPhen, I-Mutant, PROVEAN, PANTHER, SNPS\&GO and PHD-SNP are utilized. Through analyzing single nucleotide polymorphisms (SNPs) by these in silico approaches, 28 out of 285 missense SNPs were predicted to be damaging. By integrating outcomes from these in silico approaches, a prediction algorithm (sensitivity $94 \%$, specificity $80 \%$ ) was thereby developed. Three dimensional structural analysis of 5 candidate SNPs (P533R, P496R, L346R, D349G, T374P) were performed by SWISS PDB viewer, which revealed specific structural changes responsible for the functional impacts of these SNPs. Additionally, SNPs in the untranslated region were analyzed by UTRscan and PolymiRTS. Moreover, by investigating known pathogenic mutations and relevant patient phenotypes in previous publications, phenotype severity (severe, intermediate or mild) of each mutation was deduced.
\end{abstract}

Conclusions: Collectively, these results identified potential candidate SNPs with functional significance for studying MPS I disease. This study also demonstrates the effectiveness, reliability and simplicity of these in silico approaches in addressing complexity of underlying genetic basis of MPS I disease. Further, a step-by-step guideline for phenotype prediction of MPS I disease is established, which can be broadly applied in other lysosomal diseases or genetic disorders.

Keywords: In silico, Single nucleotide polymorphism, Genotype/phenotype correlation, Mucopolysaccharidosis

\section{Background}

Mucopolysaccharidosis type I (MPS I) is a lysosomal disease included within the genetically heterogeneous group of mucopolysaccharidoses (MPSs). MPS I results from mutations in the gene encoding the lysosomal enzyme $\alpha$-L-iduronidase (IDUA; glycosaminoglycan a-Liduronohydrolase, OMIM 252800) [1]. Deficiency of IDUA leads to progressive lysosomal accumulation of glycosaminoglycans (GAG) heparan and dermatan sulfate in tissues. Based on the severity of symptoms, MPS I can

\footnotetext{
* Correspondence: ouxxx045@umn.edu

1 Gene Therapy Center, Department of Pediatrics, University of Minnesota,

Minneapolis, MN 55455, USA

Full list of author information is available at the end of the article
}

be divided into three subtypes, from mild (Scheie syndrome, OMIM 607016) to intermediate (Hurler-Scheie syndrome, OMIM 607015) to severe (Hurler syndrome, OMIM 607016). Scheie or Hurler-Scheie patients have symptoms including growth delay, aortic valvular disease, skeletal dysplasias, corneal clouding and joint stiffness. In addition to having these symptoms, but in a more pronounced way, Hurler patients also have growth delay, hepatosplenomegaly, coarse facial features, hydrocephalus, mental retardation and neurodegeneration.

It has been shown that the earlier enzyme replacement therapy or hematopoetic stem cell transplantation is performed, the better the outcome is [2-5]. Since early initiation of treatment is more likely to improve clinical 
outcomes, early diagnosis and accurate phenotype prediction are essential. However, genotype/phenotype correlation of MPS I has not been well established [6, 7]. To date, assessment of the phenotype is generally based on clinical signs and symptoms. A recent study showed a lack of consensus on the assessment of phenotypic severity solely based on signs and symptoms at presentation [8]. Therefore, establishment of a reliable and easy-to-use phenotype prediction method based on genotype will be of great benefit.

The single nucleotide polymorphisms (SNPs) are the most common form of genetic mutations. SNP was originally defined as a single nucleotide variant with a frequency in genome of more than $1 \%$ [9]. In this study, for the simplicity of description, single nucleotide variants with a frequency of less than $1 \%$ were also included in the analysis. While many SNPs are phenotypically neutral, others could cause disease, predispose human to disease, or influence response to medicine. Previous studies on polymorphisms screening by in silico analysis contributed to predicting the functional non-synonymous SNPs (nsSNPs) in genes such as G6PD [10], ATM [11], PTEN [12], BRAF [13] and BUB1B [14]. This powerful computed methodology enables prioritizing SNPs with functional significance from a large quantity of neutral non-risk variants. To date, computational analyses of IDUA gene for phenotype prediction have not been performed. To this end, a number of bioinformatics tools, based on recent findings from evolutionary biology, protein structure research, machine learning and computational biology, may provide useful information for assessing the functional impacts of SNPs. A stepwise guideline for phenotype prediction based on genotype is established, which will benefit early diagnosis and proper treatment allocation for MPS I patients.

\section{Methods \\ Dataset}

The SNPs information (Protein accession number and SNP ID) of the IDUA gene was retrieved from the NCBI dbSNP (http://www.ncbi.nlm.nih.gov/snp/). Known disease-associated mutations in IDUA gene were retrieved from The Human Gene Mutation Database (http:// www.hgmd.cf.ac.uk/ac/index.php).

\section{SIFT}

SIFT (Sorting Intolerant From Tolerant; http://sift.jcvi.org/) can predict the effect of amino acid substitution on protein function, and classify it as 'tolerated' or 'deleterious' [15]. SIFT applies multiple alignment information for the query sequence and predicts whether substitutions are 'tolerated' or 'deleterious' by calculating the tolerance index score $(0$ to 1$)$. Tolerance index score is a normalized probability that an amino acid substitution is tolerated. Substitutions with a tolerance index less than 0.05 are predicted to be 'deleterious' and those with greater than or equal to 0.05 are predicted as 'tolerated'. The analysis was performed using the default settings.

\section{PolyPhen}

PolyPhen (Polymorphism Phenotyping; http://genetics.bwh. harvard.edu/pph2/) is a probabilistic classifier which predicts the functional impacts of SNPs. PolyPhen calculates position-specific independent count (PSIC) scores for every substitution and estimates the difference between the variant scores. Based on PSIC, Polyphen classifies SNPs into 'probably damaging' (score $>0.85$ ), 'possibly damaging' (score > 0.15) and 'benign' (the rest) [16].

\section{I-Mutant}

I-Mutant (http://folding.biofold.org/cgi-bin/i-mutant2.0) is a neural-network-based web server for the automatic prediction of protein stability changes upon single amino acid substitution. I-Mutant performs analyses based on the protein sequence combined with mutational position. The output is the predicted free energy change (DDG), which classifies the prediction into: 'large decrease' (DDG $<-0.5 \mathrm{kcal} / \mathrm{mol}$ ), 'large increase' (DDG $>0.5 \mathrm{kcal} /$ $\mathrm{mol})$, or 'neutral' $(-0.5<\mathrm{DDG}<0.5 \mathrm{kcal} / \mathrm{mol})$ [17].

\section{PROVEAN}

PROVEAN (Protein Variation Effect Analyzer; http:// provean.jcvi.org) is a sequence based predictor that estimates the impact of protein sequence variation on protein function [18]. In PROVEAN, BLAST hits with more than $75 \%$ global sequence identity are clustered together, and top 30 such clusters from a supporting sequence are averaged within and across clusters to generate the final score. A protein variant is predicted to be 'deleterious' if the final score is below -2.5 , and is predicted to be 'neutral' otherwise.

\section{PANTHER}

PANTHER (http://www.pantherdb.org/) is a database which contains a collection of protein families and subfamilies that predict the occurrence of an amino acid at a position in a family of evolutionarily related protein [19]. PANTHER uses hidden Markov model (HMM) based statistical modeling methods and multiple sequence alignments to perform evolutionary analysis of coding nsSNPs. By calculating the substitution position-specific evolutionary conservation score (subPSEC) based on an alignment of evolutionarily related proteins, PANTHER estimates the likelihood of a particular nsSNP causing a functional impact. Based on subPSEC scores, PANTHER classifies SNPs as 'deleterious' (score $<-3$ ) or 'neutral' (score $>-3$ ). 


\section{SNPs\&GO}

SNPs\&GO (Single Nucleotide Polymorphism Database \& Gene Ontology; http://snps.biofold.org/snps-and-go/ snps-and-go.html) is an support vector machine (SVM) based method used to predict the disease related mutations from protein sequences with a scoring accuracy of $82 \%$ and Matthews correlation coefficient of 0.63 . For SNPs\&GO, FASTA sequence of whole protein is considered to be an input option and output will be the prediction results based on the discrimination among 'disease' and 'neutral' variations of protein sequence. The probability score higher than 0.5 is defined as 'disease' [20].

\section{PHD-SNP}

PHD-SNP (Predictor of Human Deleterious Single Nucleotide Polymorphisms; http://snps.biofold.org/ phd-snp/phd-snp.html) is an SVM-based classifier, trained over a million amino acid polymorphism datasets using supervised training. PHD-SNP predicts whether the given amino acid substitution leads to 'disease' or 'neutral' along with the reliability index score [21].

\section{NetSurfP}

NetSurfP (http://www.cbs.dtu.dk/services/NetSurfP/) is a web server that predicts the surface accessibility and secondary structure of amino acids. The reliability of this NetsurfP is given in the form of Z-score. The Z-score highlights the surface prediction reliability, but not associated with secondary structure [22].

\section{Modeling of mutant protein structures}

The Swiss-PDB Viewer, a free molecular graphics program was used for viewing the modeled structures and for calculation of the root mean square deviation (RMSD) between the native and mutant structures. Swiss-PDB viewer named as Deep View, a stand-alone program, was used as an analytical tool for macromolecules [23]. To superimpose protein structures, the "Magic Interactive Fit" command was used for detection of a stretch of similar residues at sequence level to obtain a structural fit between the two models. Energy minimization for three-dimensional (3D) structures was performed using NOMAD-Ref server (http://lorentz.immstr.pasteur.fr/nomad-ref.php) [24]. Conjugate gradient method was used for energy minimization of the 3D structures.

\section{Project HOPE}

Project Have yOur Protein Explained (HOPE; http:// www.cmbi.ru.nl/hope/home) is an easy-to-use web service that analyzes the structural effects of a point mutation in a protein sequence. HOPE provides the $3 \mathrm{D}$ structural visualization of mutated proteins by using UniProt and
DAS prediction servers. HOPE server predicts the output in the form of structural variation between mutant and wild type residues [25].

\section{UTRscan}

UTRscan (http://itbtools.ba.itb.cnr.it/utrscan) is a web server that can analyze the untranslated regions (5' UTR and 3' UTR) of eukaryotic mRNA which are involved in many post-transcriptional regulatory pathways that control mRNA localization, stability and translation [26]. The internet resource for UTR analysis are UTRdb, which contains experimentally proven biological activity of functional pattern of UTR sequence from eukaryotic mRNAs. If different sequences for each UTR SNP are found to have different functional patterns, that particular UTR SNP is predicted to have functional significance.

\section{PolymiRTS}

PolymiRTS database (http://compbio.uthsc.edu/miRSNP/) was used specifically for the analysis of SNPs in the 3' UTR. The polymorphic microRNA target sites are classified into four classes [27]. Specifically, class 'D' may cause loss of normal repression, and class ' $\mathrm{C}$ ' may cause abnormal gene repression control. Therefore, these two classes of PolymiRTS are most likely to have functional impacts.

\section{Results}

\section{Analysis of missense SNPs using a combination of bioinformatics tools}

Polymorphisms in the IDUA gene were retrieved from NCBI dbSNP database. Non-synonymous SNPs (nsSNPs) from the coding region, and untranslated (5'and $3^{\prime}$ ) region were selected for further analysis. The impacts of any amino acid substitution with its functional significance and physical properties can be determined using SIFT by aligning homologous and orthologous protein sequence. A total of 285 missense SNPs of IDUA gene were analyzed using SIFT. Out of 285 SNPs, 201(71\%) were predicted to be 'deleterious' (tolerance index <0.05), while $157(55 \%)$ were 'highly deleterious' (tolerance index $=0$ ). All 201 SNPs predicted to be 'deleterious' by SIFT were further analyzed by PolyPhen. For every input SNP, Polyphen calculates PSIC score and perform BLAST query to identify homologous protein. A total of 149 SNPs were predicted to be 'probably damaging'. For further confirmation, the PolyPhen results were subjected to I-Mutant, which is a routine SNP prediction tool based on neural network, for adding another layer of confirmation. I-Mutant estimates the effect of substitution on protein stability by calculating the reliability index $\left(25^{\circ} \mathrm{C}, \mathrm{pH} 7.0\right)$. Out of 149 missense SNPs analyzed, 107 (72\%) were predicted to cause 'large decrease', while 42 were predicted to cause 'neutral 
stability'. The remaining 107 SNPs were analyzed by PROVEAN, yielding 93 deleterious and 14 neutral SNPs. Therefore, 93 out of 285 SNPs were predicted to be damaging by 4 different methods and summarized in Table 1 .

All 93 SNPs identified were further analyzed by PANTHER, SNPs\&GO and PHD-SNP. PANTHER characterizes the effect of amino acid variation on protein function via HMM based statistical modeling. PANTHER can classify proteins by function, adding another layer of complexity to refine SNP prediction. SNPs\&GO predicts the log-odd (LGO) score from the GO data base by placing the similar proteins in the same dataset. PHD-SNP is an SVM-based classifier, trained over a million amino acid polymorphism datasets using supervised training. Out of the 93 SNPs, 28 were predicted to be disease-associated by three methods (Table 2).

\section{Biophysical validation and 3D structure analysis of missense SNPs}

Based on the in silico analyses performed, 28 SNPs were selected for biophysical analysis using NetSurfP. The location and the type of a mutated residue can affect the stability of the protein by decreasing the solvent accessibility of a residue decreases. NetSurfP Z-score allows for the identification of the most reliable predictions for both buried and exposed amino acids. Out of 28 SNPs, a huge drift in the Z-score was observed for 5 SNPs (Table 3).

To analyze the 3D structural change introduced by these 5 SNPs, we performed structural analysis by comparing the native and mutant protein structures. Briefly, the native structure of IDUA was extracted from Protein Data Bank (ID 3 W81). Single amino acid substitution and superimposition of native and mutated structures were examined using Swiss-PDB viewer, and their degree of similarity was measured as the RMSD value. RMSD values between native and each mutant structure are $<0.5 \AA$, indicating a minor structural change caused by the SNP. An illustration of overall superimposition by Swiss-PDB viewer is shown in Fig. 1, while detailed structural changes in Fig. 2. Total energy values of native structure and 5 mutant structures were calculated after energy minimization by NOMAD_Ref and summarized in Table 4. The total energy of three mutant models (L346R, P496R and P533R) is significantly higher than that of the native model, indicating that the mutation decreases the protein stability.

Specifically, rs772416503 leads to conversion of proline into arginine at position 496 (P496R). The hydrophobic environment around Pro496 leaves no room for a bulky polar residue (arginine). This mutation (P496R) may interfere with the placement of Asn372 glycan over the active site, and thereby affect enzyme catalytic activity. Rs371397270 leads to conversion of aspartic acid into glycine at position 349 (D349G). Asp349 is located in triosephosphateisomerase (TIM) barrel active site and interacts with substrate. Besides, since glycine is smaller than aspartic acid, the mutation will cause an empty space in the core of the protein. The charge of the buried wild-type residue is also lost due to this mutation. Therefore, D349G will also cause loss of hydrogen bonds in the core of the protein and thereby disturb correct folding. Rs121965021 (P533R) is located in the $\beta$ sandwich. Prolines are known to have a very rigid structure, sometimes forcing the backbone in a specific conformation. P533R may disturb this special conformation and destabilize the $\beta$ sandwich domain by introducing the side chain of arginine. Besides, only the wild type residue proline is found at this position. Mutation of a $100 \%$ conserved residue is usually damaging for the protein. Rs121965033 (L346R), located in the TIM barrel, may cause steric hindrance and destabilize active site confirmation. The mutant residue (Arg) introduces a charge in a buried residue (Leu) which affects protein folding. Besides, since Leu346 is buried in the core of the protein, Arg is bigger and probably will not fit. This mutation will cause loss of hydrophobic interactions in the core of the protein. Rs775816150 (T374P) is located at Thr374, a conserved $\mathrm{N}$ glycosylation site. It has also been shown that $\mathrm{N}$-glycans are essential for substrate binding and catalytic activity of IDUA [28]. Therefore, this mutation (T374P) may lead to decrease or loss of catalytic activity of IDUA.

\section{Establishment and evaluation of SNPs prediction algorithm}

By integrating outcomes of the bioinformatics tools listed in Section 3.1, a prediction algorithm (SAAMP: Single Amino Acid Mutation Predictor) with a pathogenic index (PI) was developed. PI is defined as percentage of 'damaging' predictions from these 7 bioinformatics tools. The higher the PI is, the more pathogenic the SNP is. The cutoff value is set at 0.43 . When PI is $\geq 0.43$ (larger than or equal to 3 damaging related predictions), the mutation is defined as 'pathogenic', otherwise it is 'benign'. A total of 81 known disease-associated missense mutations and 15 known benign polymorphisms of IDUA were analyzed by these bioinformatics tools, and the PI of each mutation was calculated. By assessing false positives and false negatives, a sensitivity of $94 \%$ and a specificity of $80 \%$ were reached. The false positives and false negatives were evaluated manually, however, no significant patterns were observed. It might be due to the differences in methodologies utilized by these in silico tools. Alternatively, when the cut-off value is set as 0.57 (larger than or equal to 4 damaging related predictions), a sensitivity of 
Table 1 List of 91 nsSNP predicted as damaging by SIFT, PolyPhen, I-Mutant, PROVEAN

\begin{tabular}{|c|c|c|c|c|c|c|c|c|c|}
\hline SNP ID & AA change & SIFT & Score & PolyPhen & Score & I-Mutant & Score & PROVEAN & Score \\
\hline rs121965021 & P533R & Deleterious & 0 & Probably damaging & 1 & Large decrease & -0.75 & Deleterious & -7.1 \\
\hline rs121965029 & R89Q & Deleterious & 0 & Probably damaging & 1 & Large decrease & -0.73 & Deleterious & -3.08 \\
\hline rs121965030 & A300T & Deleterious & 0 & Probably damaging & 0.999 & Large decrease & -0.77 & Deleterious & -3.68 \\
\hline rs121965031 & R619G & Deleterious & 0 & Probably damaging & 0.999 & Large decrease & -1.51 & Deleterious & -4.63 \\
\hline rs121965033 & L346R & Deleterious & 0 & Probably damaging & 1 & Large decrease & -1.77 & Deleterious & -5.3 \\
\hline rs138731804 & A160T & Deleterious & 0 & Probably damaging & 1 & Large decrease & -0.67 & Deleterious & -3.31 \\
\hline rs140294059 & C205S & Deleterious & 0.04 & Probably damaging & 0.964 & Large decrease & -1.09 & Deleterious & -7.57 \\
\hline rs147353014 & $\mathrm{L} 237 \mathrm{H}$ & Deleterious & 0 & Probably damaging & 1 & Large decrease & -1.98 & Deleterious & -6.4 \\
\hline rs148789453 & L238Q & Deleterious & 0 & Probably damaging & 1 & Large decrease & -2.05 & Deleterious & -5.33 \\
\hline rs183347428 & D223N & Deleterious & 0 & Probably damaging & 1 & Large decrease & -1.01 & Deleterious & -3.03 \\
\hline rs200448421 & R628P & Deleterious & 0 & Probably damaging & 0.999 & Large decrease & -0.72 & Deleterious & -3.86 \\
\hline rs201268637 & R263W & Deleterious & 0 & Probably damaging & 0.995 & Large decrease & -0.71 & Deleterious & -5.39 \\
\hline rs202051939 & $\mathrm{S} 269 \mathrm{C}$ & Deleterious & 0 & Probably damaging & 1 & Large decrease & -0.62 & Deleterious & -3.94 \\
\hline rs368241547 & $\mathrm{F} 247 \mathrm{~L}$ & Deleterious & 0.02 & Probably damaging & 0.993 & Large decrease & -1.28 & Deleterious & -4.33 \\
\hline rs368454909 & D349N & Deleterious & 0 & Probably damaging & 1 & Large decrease & -0.78 & Deleterious & -4.64 \\
\hline rs369090960 & G265R & Deleterious & 0 & Probably damaging & 1 & Large decrease & -0.66 & Deleterious & -7.46 \\
\hline rs371397270 & D349G & Deleterious & 0 & Probably damaging & 1 & Large decrease & -1.08 & Deleterious & -6.43 \\
\hline rs373037758 & L256P & Deleterious & 0 & Probably damaging & 1 & Large decrease & -1.98 & Deleterious & -6.1 \\
\hline rs373342547 & F143 L & Deleterious & 0.05 & Probably damaging & 1 & Large decrease & -0.69 & Deleterious & -4.27 \\
\hline rs374699130 & A319T & Deleterious & 0 & Probably damaging & 1 & Large decrease & -0.52 & Deleterious & -3.78 \\
\hline rs374779600 & P533A & Deleterious & 0 & Probably damaging & 1 & Large decrease & -1.03 & Deleterious & -6.51 \\
\hline rs374779600 & P533S & Deleterious & 0 & Probably damaging & 1 & Large decrease & -1.18 & Deleterious & -6.51 \\
\hline rs375300630 & G244D & Deleterious & 0 & Probably damaging & 1 & Large decrease & -0.89 & Deleterious & -5.7 \\
\hline rs376573681 & $1272 \mathrm{~T}$ & Deleterious & 0 & Probably damaging & 1 & Large decrease & -2.04 & Deleterious & -4.43 \\
\hline rs398123253 & W434C & Deleterious & 0 & Probably damaging & 1 & Large decrease & -1.19 & Deleterious & -7.94 \\
\hline rs527336882 & L365 V & Deleterious & 0 & Probably damaging & 1 & Large decrease & -1.59 & Deleterious & -2.65 \\
\hline rs537047205 & D119A & Deleterious & 0 & Probably damaging & 0.993 & Large decrease & -0.6 & Deleterious & -4.43 \\
\hline rs546808806 & P377L & Deleterious & 0 & Probably damaging & 0.996 & Large decrease & -0.62 & Deleterious & -7.86 \\
\hline rs546933529 & G253C & Deleterious & 0 & Probably damaging & 1 & Large decrease & -1.21 & Deleterious & -6.19 \\
\hline rs555091763 & 1283T & Deleterious & 0 & Probably damaging & 0.996 & Large decrease & -1.76 & Deleterious & -4.22 \\
\hline rs558683362 & M133l & Deleterious & 0 & Probably damaging & 0.997 & Large decrease & -0.61 & Deleterious & -3.49 \\
\hline rs564306004 & G84S & Deleterious & 0 & Probably damaging & 1 & Large decrease & -1.25 & Deleterious & -4.42 \\
\hline rs587779401 & Y625C & Deleterious & 0 & Probably damaging & 1 & Large decrease & -1.41 & Deleterious & -5.42 \\
\hline rs74385837 & L237F & Deleterious & 0 & Probably damaging & 1 & Large decrease & -1.12 & Deleterious & -3.6 \\
\hline rs746018077 & $\mathrm{F} 495 \mathrm{~L}$ & Deleterious & 0 & Probably damaging & 0.977 & Large decrease & -0.82 & Deleterious & -4.36 \\
\hline rs746606129 & Q328H & Deleterious & 0 & Probably damaging & 1 & Large decrease & -2.16 & Deleterious & -4.27 \\
\hline rs746766617 & N348 K & Deleterious & 0 & Probably damaging & 0.994 & Large decrease & -0.64 & Deleterious & -4.96 \\
\hline rs747827435 & N350D & Deleterious & 0 & Probably damaging & 1 & Large decrease & -0.6 & Deleterious & -4.55 \\
\hline rs748239393 & F287C & Deleterious & 0 & Probably damaging & 1 & Large decrease & -1.52 & Deleterious & -6.72 \\
\hline rs748589618 & L216P & Deleterious & 0 & Probably damaging & 1 & Large decrease & -1.61 & Deleterious & -6.03 \\
\hline rs749645656 & D477G & Deleterious & 0 & Probably damaging & 0.999 & Large decrease & -1.54 & Deleterious & -4.93 \\
\hline rs750230093 & R255W & Deleterious & 0 & Probably damaging & 1 & Large decrease & -0.56 & Deleterious & -6.87 \\
\hline rs750496798 & R363C & Deleterious & 0 & Probably damaging & 1 & Large decrease & -0.82 & Deleterious & -7.39 \\
\hline rs750893089 & Р309T & Deleterious & 0 & Probably damaging & 1 & Large decrease & -1.26 & Deleterious & -5.62 \\
\hline
\end{tabular}


Table 1 List of 91 nsSNP predicted as damaging by SIFT, PolyPhen, I-Mutant, PROVEAN (Continued)

\begin{tabular}{|c|c|c|c|c|c|c|c|c|c|}
\hline rs751396984 & R383G & Deleterious & 0 & Probably damaging & 1 & Large decrease & -1.15 & Deleterious & -5.08 \\
\hline rs751547595 & $\mathrm{A} 367 \mathrm{~T}$ & Deleterious & 0 & Probably damaging & 0.976 & Large decrease & -0.92 & Deleterious & -3.39 \\
\hline rs751676744 & V88F & Deleterious & 0 & Probably damaging & 1 & Large decrease & -1.29 & Deleterious & -3.65 \\
\hline rs751792135 & G78D & Deleterious & 0 & Probably damaging & 0.998 & Large decrease & -0.96 & Deleterious & -3.77 \\
\hline rs751861062 & A204T & Deleterious & 0 & Probably damaging & 1 & Large decrease & -0.86 & Deleterious & -3.67 \\
\hline rs752529809 & P385S & Deleterious & 0 & Probably damaging & 0.997 & Large decrease & -1.37 & Deleterious & -6.99 \\
\hline rs753308650 & G168R & Deleterious & 0 & Probably damaging & 1 & Large decrease & -0.69 & Deleterious & -7.18 \\
\hline rs753875643 & P232T & Deleterious & 0 & Probably damaging & 1 & Large decrease & -1.31 & Deleterious & -6.77 \\
\hline rs753905054 & D570G & Deleterious & 0 & Probably damaging & 1 & Large decrease & -0.81 & Deleterious & -4.43 \\
\hline rs754154200 & E182K & Deleterious & 0 & Probably damaging & 1 & Large decrease & -0.88 & Deleterious & -3.76 \\
\hline rs754674352 & P128S & Deleterious & 0 & Probably damaging & 1 & Large decrease & -1.39 & Deleterious & -6.2 \\
\hline rs754681846 & R368C & Deleterious & 0 & Probably damaging & 0.976 & Large decrease & -0.81 & Deleterious & -6.8 \\
\hline rs754876066 & Т194P & Deleterious & 0 & Probably damaging & 0.999 & Large decrease & -0.96 & Deleterious & -4.7 \\
\hline rs754949360 & $\mathrm{R} 383 \mathrm{H}$ & Deleterious & 0 & Probably damaging & 1 & Large decrease & -1.12 & Deleterious & -3.8 \\
\hline rs757171895 & G208S & Deleterious & 0 & Probably damaging & 1 & Large decrease & -1.19 & Deleterious & -5.77 \\
\hline rs757706461 & P183S & Deleterious & 0 & Probably damaging & 1 & Large decrease & -1.44 & Deleterious & -7.18 \\
\hline rs758452450 & A75T & Deleterious & 0 & Probably damaging & 1 & Large decrease & -0.63 & Deleterious & -2.58 \\
\hline rs760900176 & P229L & Deleterious & 0 & Probably damaging & 0.963 & Large decrease & -0.63 & Deleterious & -5.85 \\
\hline rs762037549 & E582K & Deleterious & 0 & Probably damaging & 1 & Large decrease & -0.66 & Deleterious & -2.74 \\
\hline rs762623046 & $\mathrm{R} 166 \mathrm{~T}$ & Deleterious & 0 & Probably damaging & 0.975 & Large decrease & -0.96 & Deleterious & -4.06 \\
\hline rs764882035 & V254G & Deleterious & 0 & Probably damaging & 0.999 & Large decrease & -3.19 & Deleterious & -4.6 \\
\hline rs766030255 & T179S & Deleterious & 0 & Probably damaging & 0.957 & Large decrease & -0.58 & Deleterious & -2.96 \\
\hline rs766033352 & I259M & Deleterious & 0 & Probably damaging & 1 & Large decrease & -1.73 & Deleterious & -2.7 \\
\hline rs76722191 & V322E & Deleterious & 0 & Probably damaging & 1 & Large decrease & -0.81 & Deleterious & -5.5 \\
\hline rs768389832 & P54S & Deleterious & 0 & Probably damaging & 1 & Large decrease & -1.43 & Deleterious & -5.36 \\
\hline rs769331894 & F177 L & Deleterious & 0 & Probably damaging & 1 & Large decrease & -1.02 & Deleterious & -5.27 \\
\hline rs769805145 & P288A & Deleterious & 0 & Probably damaging & 1 & Large decrease & -1.34 & Deleterious & -7.62 \\
\hline rs770087890 & G197A & Deleterious & 0 & Probably damaging & 0.999 & Large decrease & -1.07 & Deleterious & -5.27 \\
\hline rs771733089 & $\mathrm{R} 83 \mathrm{C}$ & Deleterious & 0 & Probably damaging & 0.997 & Large decrease & -0.89 & Deleterious & -2.72 \\
\hline rs772416503 & P496R & Deleterious & 0 & Probably damaging & 1 & Large decrease & -0.7 & Deleterious & -7.58 \\
\hline rs772448566 & F352 L & Deleterious & 0 & Probably damaging & 1 & Large decrease & -1.17 & Deleterious & -5.57 \\
\hline rs772855552 & A351T & Deleterious & 0 & Probably damaging & 0.999 & Large decrease & -0.75 & Deleterious & -3.44 \\
\hline rs773471238 & V379G & Deleterious & 0 & Probably damaging & 1 & Large decrease & -2.32 & Deleterious & -5.49 \\
\hline rs773908263 & P81S & Deleterious & 0 & Probably damaging & 1 & Large decrease & -1.86 & Deleterious & -6.31 \\
\hline rs774139207 & E299D & Deleterious & 0 & Probably damaging & 1 & Large decrease & -0.77 & Deleterious & -2.95 \\
\hline rs775542391 & L114R & Deleterious & 0 & Probably damaging & 1 & Large decrease & -1.76 & Deleterious & -4.89 \\
\hline rs775816150 & T374P & Deleterious & 0 & Probably damaging & 1 & Large decrease & -0.81 & Deleterious & -5.09 \\
\hline rs776561903 & P55A & Deleterious & 0 & Probably damaging & 0.997 & Large decrease & -1.41 & Deleterious & -4.57 \\
\hline rs780165694 & Y76C & Deleterious & 0 & Probably damaging & 1 & Large decrease & -1.09 & Deleterious & -4.34 \\
\hline rs781136336 & L526P & Deleterious & 0 & Probably damaging & 0.999 & Large decrease & -1.11 & Deleterious & -3.93 \\
\hline rs781149866 & $\mathrm{R} 368 \mathrm{H}$ & Deleterious & 0 & Probably damaging & 1 & Large decrease & -1.17 & Deleterious & -3.47 \\
\hline rs794726877 & G51D & Deleterious & 0 & Probably damaging & 1 & Large decrease & -0.59 & Deleterious & -5.45 \\
\hline rs794727017 & P510R & Deleterious & 0 & Probably damaging & 1 & Large decrease & -0.62 & Deleterious & -5.79 \\
\hline rs794727896 & T388 K & Deleterious & 0 & Probably damaging & 1 & Large decrease & -0.74 & Deleterious & -4.24 \\
\hline rs866224971 & R447C & Deleterious & 0 & Probably damaging & 0.999 & Large decrease & -1.12 & Deleterious & -3.3 \\
\hline
\end{tabular}


Table 1 List of 91 nsSNP predicted as damaging by SIFT, PolyPhen, I-Mutant, PROVEAN (Continued)

\begin{tabular}{llllllllll}
\hline rs869025584 & L218P & Deleterious & 0 & Probably damaging & 0.996 & Large decrease & -1.63 & Deleterious & -5.03 \\
rs875989946 & W175R & Deleterious & 0 & Probably damaging & 1 & Large decrease & -1.23 & Deleterious & -13.14 \\
rs375819348 & P493R & Deleterious & 0 & Probably damaging & 1 & Large decrease & -0.82 & Deleterious & -8.33 \\
rs767140903 & P302R & Deleterious & 0 & Probably damaging & 1 & Large decrease & -0.95 & Deleterious & -7.79 \\
\hline
\end{tabular}

$79 \%$ and a specificity of $93 \%$ was calculated. In order to increase the probability of identifying pathogenic mutations and minimize the risk of neglecting patients, high sensitivity is preferable and the cut-off value of 0.43 is recommended.

\section{Functional SNPs in UTRs identified by UTSscan and PolymiRTs}

All of the 177 UTR SNPs were analyzed using UTRscan. It has been shown that polymorphisms in 3' UTR region can affect the gene expression pattern during mRNA translation, while the polymorphisms in 5' UTR region affect the RNA half-life by altering the polyadenylation $[28,29]$. After comparing the functional elements for each UTR SNP, we predicted that 6 SNPs in $5^{\prime}$ UTR are related to the functional pattern changes including internal ribosome entry site (IRES) and 15-Lipoxygenase Differentiation Control Element (15-LOX-DICE) (Table 5). The IRES is involved in internal mRNA ribosome binding, which allows for translation when the

Table $\mathbf{2}$ List of 28 nsSNP predicted as associated with disease by PHD-SNP, PANTHER and SNP\&GO

\begin{tabular}{|c|c|c|c|c|c|c|c|}
\hline SNP ID & AA change & PHD-SNP & Probability & PANTHER & Probability & SNP\&GO & Probability \\
\hline rs76722191 & V322E & Disease & 0.718 & Disease & 0.712 & Disease & 0.716 \\
\hline rs121965021 & P533R & Disease & 0.635 & Disease & 0.827 & Disease & 0.671 \\
\hline rs121965029 & R89Q & Disease & 0.841 & Disease & 0.609 & Disease & 0.745 \\
\hline rs121965033 & L346R & Disease & 0.76 & Disease & 0.792 & Disease & 0.663 \\
\hline rs148789453 & L238Q & Disease & 0.777 & Disease & 0.553 & Disease & 0.513 \\
\hline rs200448421 & R628P & Disease & 0.817 & Disease & 0.529 & Disease & 0.634 \\
\hline rs368454909 & D349N & Disease & 0.723 & Disease & 0.603 & Disease & 0.54 \\
\hline rs369090960 & G265R & Disease & 0.12 & Disease & 0.841 & Disease & 0.63 \\
\hline rs371397270 & D349G & Disease & 0.754 & Disease & 0.663 & Disease & 0.593 \\
\hline rs373037758 & L256P & Disease & 0.881 & Disease & 0.795 & Disease & 0.801 \\
\hline rs374779600 & P533S & Disease & 0.539 & Disease & 0.718 & Disease & 0.579 \\
\hline rs587779401 & Y625C & Disease & 0.722 & Disease & 0.867 & Disease & 0.724 \\
\hline rs748239393 & F287C & Disease & 0.668 & Disease & 0.862 & Disease & 0.628 \\
\hline rs750496798 & R363C & Disease & 0.764 & Disease & 0.903 & Disease & 0.701 \\
\hline rs753308650 & G168R & Disease & 0.91 & Disease & 0.841 & Disease & 0.846 \\
\hline rs754154200 & E182K & Disease & 0.772 & Disease & 0.591 & Disease & 0.702 \\
\hline rs754876066 & T194P & Disease & 0.747 & Disease & 0.732 & Disease & 0.628 \\
\hline rs757706461 & P183S & Disease & 0.619 & Disease & 0.718 & Disease & 0.602 \\
\hline rs762623046 & R166T & Disease & 0.739 & Disease & 0.547 & Disease & 0.639 \\
\hline rs772416503 & P496R & Disease & 0.606 & Disease & 0.827 & Disease & 0.568 \\
\hline rs773908263 & P81S & Disease & 0.725 & Disease & 0.688 & Disease & 0.667 \\
\hline rs775542391 & L114R & Disease & 0.818 & Disease & 0.786 & Disease & 0.743 \\
\hline rs775816150 & T374P & Disease & 0.771 & Disease & 0.732 & Disease & 0.538 \\
\hline rs780165694 & Y76C & Disease & 0.646 & Disease & 0.703 & Disease & 0.522 \\
\hline rs794726877 & G51D & Disease & 0.74 & Disease & 0.79 & Disease & 0.704 \\
\hline rs875989946 & W175R & Disease & 0.866 & Disease & 0.839 & Disease & 0.831 \\
\hline rs767140903 & P302R & Disease & 0.673 & Disease & 0.827 & Disease & 0.678 \\
\hline rs375819348 & P493R & Disease & 0.681 & Disease & 0.827 & Disease & 0.633 \\
\hline
\end{tabular}


Table 3 Surface accessibility of native and mutant IDUA variants that are selected for structural analysis

\begin{tabular}{llcllll}
\hline SNP ID & AA & AA position & RSA & ASA & Z-fit score for RSA prediction & Class assignment \\
\hline rs121965021 & P & 533 & 0.341 & 48.43 & -1.149 & Buried \\
rs371397270 & R & & 0.344 & 78.73 & -0.651 & Buried \\
& D & 349 & 0.241 & 34.699 & -0.488 & Buried \\
rs775816150 & G & & 0.392 & 30.819 & -1.405 & Exposed \\
& T & \multirow{3}{*}{374} & 0.095 & 13.149 & -1.852 & Buried \\
rs772416503 & P & & 0.233 & 33.134 & -1.134 & Buried \\
& P & 496 & 0.105 & 14.928 & 0.247 & Buried \\
rs121965033 & R & & 0.164 & 37.533 & -0.253 & Buried \\
& L & \multirow{3}{*}{346} & 0.031 & 5.603 & 0.342 & Buried \\
R & & 0.108 & 24.09 & -0.954 & Buried
\end{tabular}

$A A$ amino acid, $R S A$ relative surface accessibility, $A S A$ absolute surface accessibility

conventional mechanism of translation is ineffective. 15-LOX-DICE is a multifunctional cis-element found in the 3' UTR of numerous eukaryotic mRNAs. 15LOX-DICE binds heterogeneous nuclear ribonucleoproteins (hnRNP) $\mathrm{E}$ and $\mathrm{K}$, thus mediating mRNA stabilization and translational control. Among 19 SNPs in 3' UTR region of IDUA gene, only one SNP (rs733349) is predicted to disrupt 13 non-conserved miRNA sites (ancestral allele with support <2) and create 8 new miRNA sites (Table 6).

\section{Phenotypic severity prediction of known disease-associated mutations}

Proper and timely treatment allocation based on phenotype severity prediction is essential for benefits of patients. The aforementioned bioinformatics tools are not designed specifically for MPS I disease, and are unable to predict the phenotype severity (Hurler, Hurler-Scheie or Scheie). Therefore, an extensive review of previous publications reporting pathogenic mutations of IDUA was conducted to make inferences about phenotype severity. A total of 185 mutations have been identified, including 86 missense mutations, 22 nonsense mutations, 45 deletions/insertions and 32 splicing mutations. By analyzing the phenotypes and mutations on both alleles of patients from the original reports, phenotype prediction of each mutation was conducted manually. Four general assumptions were used as followed: 1) only when both alleles are predicted to be severe, the phenotype is Hurler; 2) if one allele is predicted to be mild (intermediate) while the other severe, the phenotype is Scheie (Hurler-Scheie); 3) if both alleles are intermediate, the phenotype is Hurler-Scheie or Scheie; 4) even only one allele is predicted to be mild, the phenotype is Scheie (illustrated in Additional file 1: Fig. S1). Further, the crystal structure of IDUA has been elucidated $[30,31]$, which was used to further confirm and rectify the predictions made in Tables 7 and 8. Notably, due to lack of enough information and consensus of phenotype severity, it is difficult to make a comprehensive evaluation of reliability of the original reports. Therefore, we highlighted the severity predictions with relatively low
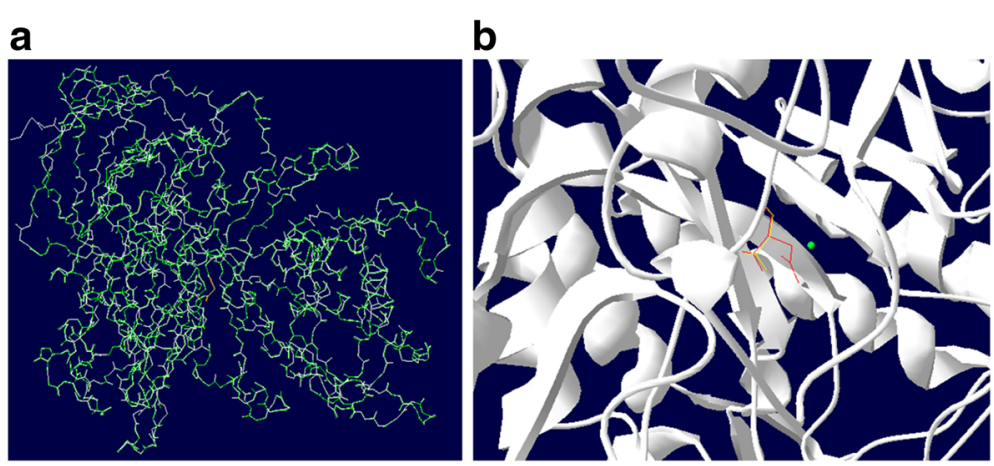

Fig. 1 Superimposed structure of native protein with modeled mutant protein for D349G. a Overall structure of the superimposed model. Native protein in white (cartoon shape), mutant protein in green, wild type residue (Asp349) in red, and mutated residue (Gly349) in yellow. b close-up view of the superimpose model. Main protein backbone in white, wild type reside (Asp349) in red, mutated residue (Gly349) in yellow, a chloride anion in green 


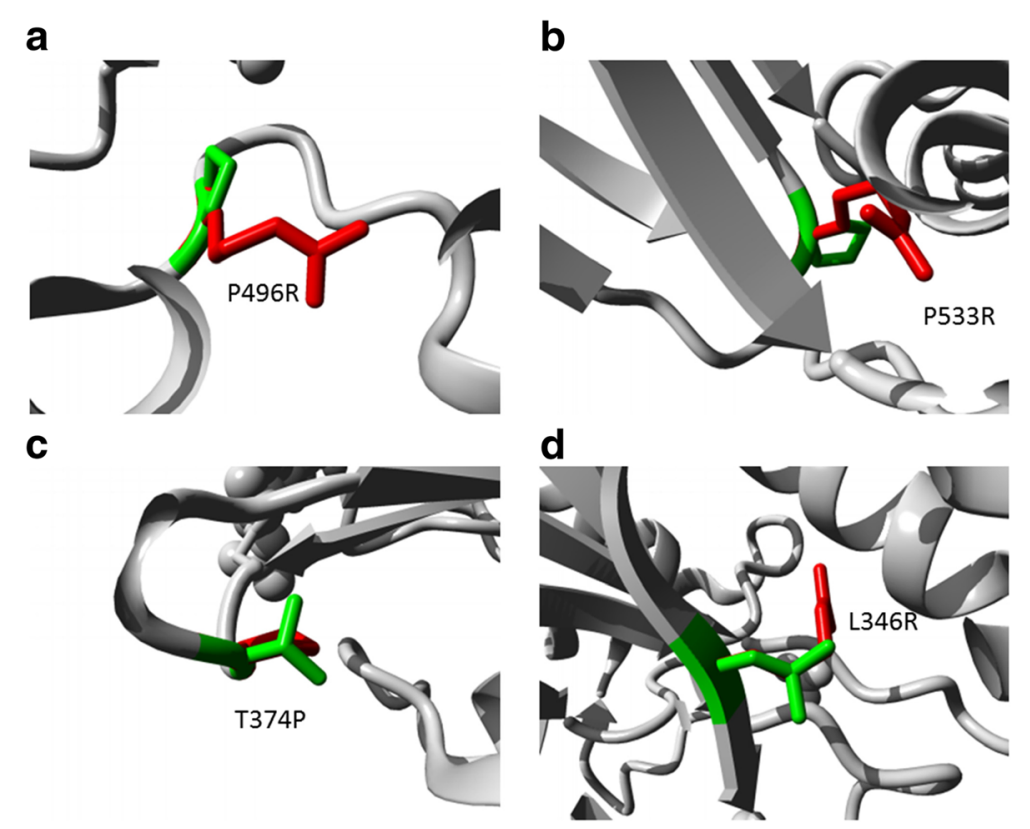

Fig. 2 Close-up view of superimposed structure of native and mutant residues (a P496R; b P533R; c T374P; $\mathbf{d}$ L346R). The main protein core is shown in white color while the wild type and mutated residues are shown in red and yellow color, respectively

reliability with ' $"$ ' in Tables 7 and 8 . All identified nonsense mutations are severe. W402X and Q70X are the most common nonsense mutations found in patients from different ethnic groups. Out of 32 splicing mutations, 20 are predicted to be severe, 5 intermediate, 1 mild (IVS5$7 \mathrm{G}>\mathrm{A}$ ) and 4 with unknown effects. Out of 45 deletions/ insertions, 38 are predicted to be severe, which is reasonable due to the usual consequence of frame shift. However, there might be some exceptions: 396insAC, c.1593delG, and 1995del11 with Hurler-Scheie or Scheie phenotype. 1995del11 is in the final exon of IDUA, which may lead to residual enzyme activity. c.1593delG was found to be in trans with a missense mutation (deduced to be severe from multiple reports) in a Hurler-Scheie patient [32]. However, although this patient is defined as Hurler-Scheie, delayed mental development was observed. Therefore, this patient may actually have Hurler disease, which will make 1592delG 'severe'. Similarly, additional evidence is

Table 4 Total energy of native and mutant structures after energy minimization

\begin{tabular}{lll}
\hline SNP ID & AA change & Total energy after minimization $(\mathrm{KJ} / \mathrm{mol})$ \\
\hline & Native & $-58,850$ \\
rs121965033 & L346R & $-57,705$ \\
rs772416503 & P496R & $-54,038$ \\
rs121965021 & P533R & $-22,157$ \\
rs775816150 & T374P & $-58,766$ \\
rs371397270 & D349G & $-58,730$ \\
\hline
\end{tabular}

required to determine the phenotypic severity of 396insAC. Missense mutations are the least severe type, with only 31 out of 86 are predicted to be severe. P533R is the most frequent but complicated missense mutation, which has been found in the homozygous state in patients with Hurler, Hurler-Scheie and Scheie phenotypes. Due to convenience consideration, the nomenclature of mutations in this study still uses the old names as reported in previous publications. However, as suggested in the current guideline on nomenclature [33], it will be important to follow this guideline to name newly identified mutations.

\section{Discussion}

The identification of SNPs responsible for specific phenotypes with molecular approaches can be expensive and time-consuming [34]. Therefore, computational approaches can be of great help by narrowing down the number of missense mutations to be screened in genetic association studies and advancing the understanding of functional and structural aspects of the protein. Since existing in silico methods have widely varying performance, no single method could be considered as the best and most accurate for predicting functional SNPs. Therefore, a combination of methods based on evolutionary information, protein structure and functional parameters were used in order to increase the prediction accuracy. Notably, there is no specific order for using these bioinformatics tools. 
Table $\mathbf{5}$ List of mRNA UTR SNPs that were predicted to be of functional significance by UTRscan server

\begin{tabular}{llll}
\hline SNP ID & Nucleotide change & UTR position & Functional element change \\
\hline rs577729544 & G/A & $5^{\prime}$ & IRES $\rightarrow$ no pattern \\
rs200237798 & G/A & $5^{\prime}$ & IRES $\rightarrow$ no pattern \\
rs372934646 & C/A & $5^{\prime}$ & IRES $\rightarrow$ no pattern \\
rs530362790 & G/A & $5^{\prime}$ & No pattern $\rightarrow$ 15-LOX-DICE \\
rs765255638 & G/T & $5^{\prime}$ & IRES $\rightarrow$ no pattern \\
rs775542391 & T/G & $5^{\prime}$ & IRES $\rightarrow$ no pattern \\
\hline
\end{tabular}

15-LOX-DICE 15-Lipoxygenase Differentiation Control Element, IRES internal ribosome entry site

In this study, significant concordance was observed between the functional consequences of nsSNPs predicted by various combinations of the tools. Out of 201 missense nsSNPs predicted to be 'deleterious' by SIFT, 149 (74\%) were also predicted to be 'probably damaging' by PolyPhen. Out of 285 missense nsSNPs, 93 (47\%) were predicted to be 'damaging' by SIFT, PolyPhen, IMutant and PROVEAN. Then, these 93 nsSNPs were analyzed by PHD-SNP, SNPs\&GO and PANTHER, and $28(30 \%)$ were predicted to be disease-associated. Further, the SNPs predicted by these in silico approaches were well supported by experimental and clinical reports. We cross-referenced the results of in silico analysis and previously identified disease-associated mutations in HGMD. Out of 28 missense SNPs (Table 2) predicted, 18 (64\%) have been identified to be diseaseassociated in the HGMD. These results demonstrated that implementations of different algorithms could serve as reliable and powerful tools for prioritizing candidate functional nsSNPs.

Based on the results in this study, a step-by-step guiding model for phenotype prediction of MPS I disease was established (Fig. 3). When a mutation is identified, 1 ) if it is a known disease-associated mutation, refer to Tables 7 and 8 for phenotype severity prediction; 2) if not, conduct the in silico analysis of coding region SNPs and UTR SNPs, respectively. As discussed previously [35], even multiple lines of computational evidence only count as a single supporting criterion for classifying variants as pathogenic or benign. Therefore, further confirmation should be conducted through biochemical and/or clinical analyses. This model will be of great use by providing a valid, time-saving, cheap and easy-to-use method for phenotype prediction for a variety of diseases including MPS I. Admittedly, there are some limitations of this model. First, the in silico analysis is not sensitive enough for phenotype severity prediction because there are no algorithms specifically designed for this purpose. Second, the 3D structural analysis relies on the availability of 3D structure, rendering it difficult for analyzing proteins without solved structures. In this case, homology modeling can be applied to bridge this gap by predicting unknown protein structures.

Table 6 Prediction result of PolymiRTS database

\begin{tabular}{|c|c|c|c|c|}
\hline SNP ID & $\mathrm{miR} I \mathrm{D}$ & Conservation & miRSite & Function class \\
\hline \multirow[t]{13}{*}{ rs733349 } & hsa-miR-128-3p & 1 & ggctgCACTGTGC & $N$ \\
\hline & hsa-miR-148a-3p & 1 & ggcTGCACTGtgc & N \\
\hline & hsa-miR-148b-3p & 1 & ggcTGCACTGtgc & $\mathrm{N}$ \\
\hline & hsa-miR-152-3p & 1 & ggcTGCACTGtgc & $\mathrm{N}$ \\
\hline & hsa-miR-216a-3p & 1 & ggctgCACTGTGC & $\mathrm{N}$ \\
\hline & hsa-miR-3681-3p & 1 & ggctgCACTGTGC & $\mathrm{N}$ \\
\hline & hsa-miR-3944-5p & 1 & gGCTGCACtgtgc & $\mathrm{N}$ \\
\hline & hsa-miR-7156-3p & 1 & GGCTGCActgtgC & $\mathrm{N}$ \\
\hline & hsa-miR-4436b-3p & 1 & ggCTGCCCTgtgc & C \\
\hline & hsa-miR-4632-5p & 1 & ggCTGCCCTgtgc & C \\
\hline & hsa-miR-6735-5p & 1 & ggCTGCCCTgtgc & C \\
\hline & hsa-miR-6879-5p & 1 & ggCTGCCCTgtgc & C \\
\hline & hsa-miR-7843-5p & 1 & ggCTGCCCTgtgc & C \\
\hline
\end{tabular}

Conservation: Occurrence of the miRNA site in other vertebrate genomes in addition to the query genome. By clicking the hyperlink, the users can examine the genomes in which this miRNA target site occurs. miRSite: Bases complementary to the seed region are in capital letters. Explanation of the function class is listed as followed. D: The derived allele disrupts a conserved miRNA site (ancestral allele with support $>=2$ ). N: The derived allele disrupts a nonconserved miRNA site (ancestral allele with support <2). C: The derived allele creates a new miRNA site. O: The ancestral allele cannot be determined 
Table 7 Phenotype/genotype correlation of missense and nonsense mutations in IDUA gene

\begin{tabular}{|c|c|c|c|c|c|}
\hline Mutation & Phenotype prediction & Mutation & Phenotype prediction & Mutation & Phenotype prediction \\
\hline Y76C & mild & M504T & intermediate & V620F & severe \\
\hline R89W & mild & L535F & intermediate & R628P & severe \\
\hline R89Q & mild, intermediate & R619G & intermediate & X654C & severe \\
\hline A160D & mild, intermediate & W626R & intermediate & L421P & unknown \\
\hline C205Y & mild & X654G & intermediate & L578Q & unknown \\
\hline G219E & mild, intermediate & X654R & intermediate, severe & G168 V & unknown \\
\hline $\mathrm{H} 240 \mathrm{R}$ & mild & $\mathrm{M} 1 \mathrm{~T}$ & severe & F52 L & unknown \\
\hline E276K & mild, intermediate & G51D & severe & L396P & unknown \\
\hline W306 L & mild, intermediate & A75T & severe & P533R & unknown \\
\hline A319V & mild, intermediate & Т103P & severe & H33P & unknown \\
\hline L346R & mild, intermediate & M133I & severe & A79V & unknown \\
\hline N348 K & mild $^{a}$ & $\mathrm{~T} 141 \mathrm{~S}$ & severe & G197S & unknown \\
\hline N350l & mild, intermediate & F177S & severe & W41X & severe \\
\hline Q380R & mild, intermediate & E182D & severe & C53X & severe \\
\hline $\mathrm{R} 383 \mathrm{H}$ & mild, intermediate & E182K & severe & Q60X & severe \\
\hline T388R & mild & P183R & severe & Q63X & severe \\
\hline S423R & mild, intermediate & D203N & severe & Y64X & severe \\
\hline R492P & mild & G208D & severe & Q70X & severe \\
\hline S633 L & mild, intermediate & G208 V & severe & Y167X & severe \\
\hline M1I & intermediate & L218P & severe & Y201X & severe \\
\hline A75P & intermediate & L237R & severe & $E 274 X$ & severe \\
\hline $\mathrm{H} 82 \mathrm{P}$ & intermediate & L238R & severe & E299X & severe \\
\hline G84R & intermediate $^{a}$ & I270S & severe & Q310X & severe \\
\hline E178K & intermediate & L308P & severe & Y343X & severe \\
\hline T179R & intermediate, severe & D315Y & severe & W402X & severe \\
\hline F188 L & intermediate $^{a}$ & A327P & severe & E404X & severe \\
\hline G197D & intermediate & D349N & severe & W420X & severe \\
\hline L238Q & intermediate & D349Y & severe & Q561X & severe \\
\hline S260F & intermediate $^{a}$ & R363C & severe & Y581X & severe \\
\hline G265R & intermediate & Т366Р & severe & Q584X & severe \\
\hline $\mathrm{R} 363 \mathrm{H}$ & intermediate & T374 N & severe & R619X & severe \\
\hline T364 M & intermediate & P385R & severe & R621X & severe \\
\hline A436P & intermediate & R489P & severe & W626X & severe \\
\hline G409R & severe & P496R & severe & R628X & severe \\
\hline L490P & intermediate & P533L & severe & & \\
\hline P496L & intermediate & F602I & severe & & \\
\hline
\end{tabular}

${ }^{a}$ was added to predictions with relatively low reliability

\section{Conclusions}

In conclusion, structural and functional impacts of nsSNPs in the IDUA gene were predicted using powerful computational tools. By predicting the possible deleterious SNPs of IDUA gene, the number of SNPs screened in association with diseases can be narrowed down to those that are most likely to alter gene function. Further, a model of phenotype prediction for MPS I disease by a combination of bioinformatics tools is established, which will benefit diagnosis and treatment allocation of MPS I patients. In the future, it will be essential to optimize the SAAMP algorithm by integrating the scores from each method with more sophisticated statistical methods, and validate it in a broad array of genes. 
Table 8 Phenotype/genotype correlation of splicing, deletions and insertions mutations in IDUA gene

\begin{tabular}{|c|c|c|c|c|c|}
\hline Mutation & Phenotype prediction & Mutation & Phenotype prediction & Mutation & Phenotype prediction \\
\hline 134del12 & severe & c.1147dupG & severe & IVS4-1G > A & intermediate, severe \\
\hline 153delC & severe & c.1166_1171dup & severe & IVS4 + $1 \mathrm{G}>\mathrm{A}$ & intermediate \\
\hline 229del3 & severe & c.1190-1delG & severe & IVS5-7G > A & mild \\
\hline 252insC & severe & c.1225dupG & severe & IVS5 + 1G > A & severe \\
\hline c.349delT & unknown & c.1244-1271del27 & severe & IVS6 + 1G >C & severe \\
\hline 396insAC & mild $^{a}$ & 1251 delC & severe & IVS6 + 1G > T & severe \\
\hline 468del3 & severe & 1277 ins 9 & severe & IVS7-4G > A & severe \\
\hline 486del6 & unknown & 1352delG & severe & IVS7 $+2 \mathrm{~T}>\mathrm{C}$ & unknown \\
\hline c.574delT & severe & c.1398delC & severe & IVS8-1G > A & severe \\
\hline 628del5 & severe & c.1589insGC & severe & IVS8 + 4G > A & intermediate \\
\hline c.657dupG & severe & c.1593delG & intermediate $^{a}$ & IVS8 $+5 G>A$ & intermediate, severe \\
\hline 668insGCG & severe & 1702delG & severe & IVS9 + 1G > T & severe \\
\hline 682insAC & severe & 1783del11 & severe & IVS9 $+2 \mathrm{~T}>\mathrm{G}$ & unknown \\
\hline 702ins10del22 & severe & c.1805delTinsGAACA & severe & IVS11-G > T & severe \\
\hline 704 ins5 & severe & 1839del29 & severe & IVS11 + 5G > A & severe \\
\hline 740delC & severe & 1902del2 & severe & IVS11 + 5G >C & severe \\
\hline 747delG & severe & c.1918_1927del10 & intermediate $^{a}$ & 3308del12 & intermediate \\
\hline 755del5 & severe & 1995del11 & intermediate $^{a}$ & IVS12 + 1G > A & severe \\
\hline c.826_828del3 & severe & D444/445 & mild & $\operatorname{IVS} 12+2 \mathrm{~T}>\mathrm{G}$ & severe \\
\hline c.854delC & severe & C. $1-2 C>G$ & severe & IVS12 + 2T > A & unknown \\
\hline c.883dupC & severe & IVS2-1G >C & severe & IVS12 + 3G >C & severe \\
\hline c.956_972 + 9delinsTA & severe & IVS2-3C > G & unknown & IVS12 + 4C > T & intermediate \\
\hline 964delC & severe & IVS2 + 1G > A & intermediate & IVS12 + 5G > A & severe \\
\hline 974ins12 & mild & IVS2 $+6 C>T$ & severe & IVS12 + 5G >C & unknown \\
\hline c.1045_1047del3 & severe & IVS3-2A $>G$ & severe & IVS12 + 6 T > A & severe \\
\hline 1132del6 & severe & IVS3 + 1G > A & severe & & \\
\hline
\end{tabular}

${ }^{a}$ was added to predictions with relatively low reliability

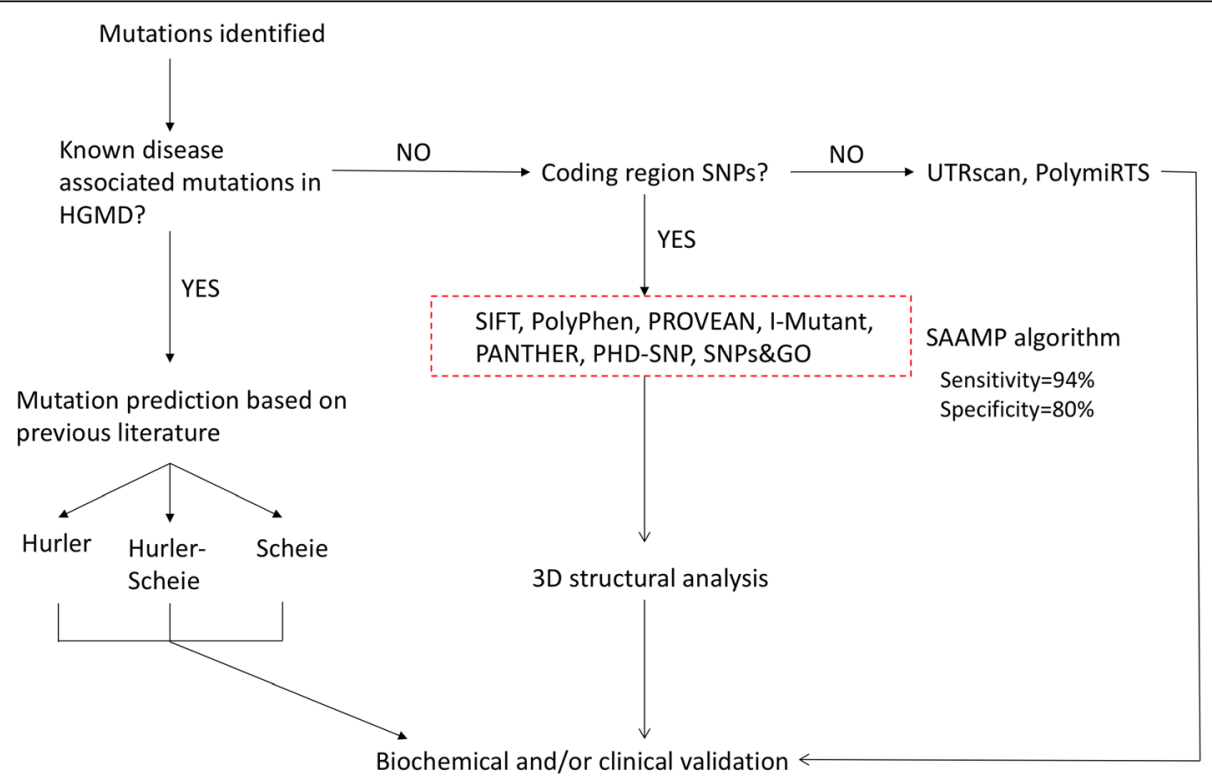

Fig. 3 Step-by-step guideline for phenotype prediction by in silico analysis 


\section{Additional file}

Additional file 1: Figure S1. General assumptions for phenotype severity prediction. (PPTX $245 \mathrm{~kb}$ )

\section{Abbreviations}

15-LOX-DICE: 15-Lipoxygenase Differentiation Control Element;

GAG: glycosaminoglycans; HGMD: human gene mutation database; HMM: hidden Markov model; hnRNP: heterogeneous nuclear ribonucleoproteins; HOPE: Have yOur Protein Explained; IDUA: a-L-iduronidase; IRES: internal ribosome entry site; MPS I: mucopolysaccharidosis type I; nsSNPs: non-synonymous SNPs; PHD-SNP: Predictor of Human Deleterious Single Nucleotide Polymorphisms; PI: pathogenic index; PolyPhen: Polymorphism Phenotyping; PROVEAN: Protein Variation Effect Analyzer; RMSD: root mean square deviation; SAAMP: Single Amino Acid Mutation Predictor; SIFT: Sorting Intolerant From Tolerant; SNPs: single nucleotide polymorphisms; SNPS\&GO: Single Nucleotide Polymorphism Database \& Gene Ontology; subPSEC: substitution position-specific evolutionary conservation score; SVM: support vector machine; TIM: triosephosphateisomerase

\section{Acknowledgements}

Not applicable.

\section{Funding}

This work is supported by NIH grant P01HD032652. Dr. Li Ou is a fellow of the Lysosomal Disease Network (U54NS065768). The Lysosomal Disease Network is a part of the Rare Diseases Clinical Research Network (RDCRN), an initiative of the Office of Rare Diseases Research (ORDR), and NCATS. This consortium is funded through a collaboration between NCATS, the National Institute of Neurological Disorders and Stroke (NINDS), and the National Institute of Diabetes and Digestive and Kidney Diseases (NIDDK).

\section{Availability of data and materials}

All data generated or analyzed during this study are included in this published article and its supplementary information files.

\section{Authors' contributions}

LO designed the study, conducted data analysis and wrote the manuscript. MJP participates in data analysis and manuscript revisions. CBW participates in the study design and manuscript revisions. All authors agree to publish this article. All authors read and approved the final manuscript.

\section{Ethics approval and consent to participate}

Not applicable.

\section{Consent for publication}

Not applicable.

\section{Competing interests}

The authors declare that they have no competing interests.

\section{Publisher's Note}

Springer Nature remains neutral with regard to jurisdictional claims in published maps and institutional affiliations.

\section{Author details}

${ }^{1}$ Gene Therapy Center, Department of Pediatrics, University of Minnesota, Minneapolis, MN 55455, USA. ²Department of Genetics, Cell Biology and Development, University of Minnesota, Minneapolis, MN 55455, USA.

Received: 17 April 2017 Accepted: 27 June 2017

Published online: 04 July 2017

\section{References}

1. Hopwood JJ, Morris CP. The mucopolysaccharidoses. Diagnosis, molecular genetics and treatment. Mol Biol Med. 1990;7:381-404.

2. Dierenfeld AD, McEntee MF, Vogler CA, Vite $\mathrm{CH}$, Chen $\mathrm{AH}$, Passage $\mathrm{M}$, et al. Replacing the enzyme alpha-L-iduronidase at birth ameliorates symptoms in the brain and periphery of dogs with mucopolysaccharidosis type I. Sci Transl Med. 2010;2:60ra89.
3. Boelens JJ, Aldenhoven M, Purtill D, Ruggeri A, Defor T, Wynn R, et al. Outcomes of transplantation using various hematopoietic cell sources in children with Hurler syndrome after myeloablative conditioning. Blood. 2013;121:3981-7.

4. Aldenhoven M, Wynn RF, Orchard PJ, O'Meara A, Veys P, Fischer A, et al. Long-term outcome of Hurler syndrome patients after hematopoietic cell transplantation: an international multicenter study. Blood. 2015;125:2164-72.

5. Sands MS, Vogler C, Kyle JW, Grubb JH, Levy B, Galvin N, et al. Enzyme replacement therapy for murine mucopolysaccharidosis type VII. J Clin Invest. 1994:93:2324-31.

6. Beesley CE, Meaney CA, Greenland G, Adams V, Vellodi A, Young EP, et al. Mutational analysis of 85 mucopolysaccharidosis type I families: frequency of known mutations, identification of 17 novel mutations and in vitro expression of missense mutations. Hum Genet. 2001:109:503-11.

7. Li P, Wood T, Thompson JN. Diversity of mutations and distribution of single nucleotide polymorphic alleles in the human alpha-L-iduronidase (IDUA) gene. Genet Med. 2002;4:420-6.

8. De Ru MH, Teunissen OGA, Van der Lee JH, Beck M, Bodamer OA, Clarke LA et al. Capturing phenotypic heterogeneity in MPS I: results of an international consensus procedure. Orphanet J Rare Dis. 2012;7:22.

9. Cargill M, Altshuler D, Ireland J, Sklar P, Ardlie K, Patil N, et al. Characterization of single nucleotide polymorphisms in coding regions of human genes. Nat Genet. 1999;22:231-8.

10. Rajith B, Geogre Priya Doss C. Path to facilitate the prediction of functional amino acid substitutions in red blood cell disorders-a computational approach. PLoS One. 2011;6:e24607.

11. Geogre Priya Doss C, Rajith B. Computational refinement of functional single nucleotide polymorphisms associated with ATM gene. PLoS One. 2012;7:e34573.

12. Geogre Priya Doss C, Rajith B. A new insight into structural and functional impact of single-nucleotide polymorphisms in PTEN gene. Cell Biochem Biophys. 2013:66:249-63.

13. Hussain MR, Shaik NA, Al-Aama JY, Asfour HZ, Khan FS, Masoodi TA, et al. In silico analysis of single nucleotide polymorphisms (SNPS) in human BRAF gene. Gene. 2012;508:188-96.

14. Akhoundi F, Parvaneh N, Modjtaba EB. In silico analysis of deleterious single nucleotide polymorphisms in human BUB1 mitotic checkpoint serine/ threonine kinase B gene. Meta Gene. 2016;28(9):142-50.

15. Ng PC, Henikoff S. Predicting deleterious amino acid substitutions. Genome Res. 2001;11:863-74.

16. Adzhubei IA, Schmidt S, Peshkin L, Ramensky VE, Gerasimova A, Bork P, et al. A method and server for predicting damaging missense mutations. Nat Methods. 2010;7:248-9.

17. Bava KA, Gromiha MM, Uedaira H, Kitajima K, Sarai A. ProTherm, version 4.0: thermodynamic database for proteins and mutants. Nucleic Acids Res. 2004:32:D120-1.

18. Choi Y, Sims GE, Murphy S, et al. Predicting the functional effect of amino acid substitutions and indels. PLoS One. 2012;7(10):e46688.

19. Mi H, Guo N, Kejariwal A, Miller JR, Chan AP. PANTHER version 6: protein sequence and function evolution data with expanded representation of biological pathways. Nucleic Acids Res. 2007:35:D247-52.

20. Calabrese R, Capriotti E, Fariselli P, Martelli PL, Casadio R. Functional annotations improve the predictive score of human disease-related mutations in proteins. Hum Mutat. 2009;30:1237-44.

21. Capriotti E, Fariselli P, Casadio R. I-Mutant2.0: predicting stability changes upon mutation from the protein sequence or structure. Nucleic Acids Res. 2005;33:W306-10.

22. Petersen B, Petersen TN, Andersen P, Nielsen M, Lundegaard C. A generic method for assignment of reliability scores applied to solvent accessibility predictions. BMC Struct Biol. 2009;9:51.

23. Guex N, Peitsch MC, Schwede T. Automated comparative protein structure modeling with SWISS-MODEL and Swiss-PdbViewer: A historical perspective. Electrophoresis. 2009:30:S162-73.

24. Lindahl E, Azuara C, Koehl P, Delarue M. NOMAD-Ref: visualization, deformation and refinement of macromolecular structures based on all-atom normal mode analysis. Nucleic Acids Res. 2006:34:W52-6.

25. Venselaar H, Te Beek TA, Kuipers RK, Hekkelman ML, Vriend G. Protein structure analysis of mutations causing inheritable diseases. An e-Science approach with life scientist friendly interfaces. BMC Bioinformatics. 2010:11:548.

26. Grillo G, Turi A, Licciulli F, Mignone F, Liuni S, Banfi S, et al. UTRdb and UTRsite (RELEASE 2010): a collection of sequences and regulatory motifs of the untranslated regions of eukaryotic mRNAs. Nucleic Acids Res. 2010;38:D75-80. 
27. Bhattacharya A, Ziebarth JD, Cui Y. PolymiRTS Database 3.0: linking polymorphisms in microRNAs and their target sites with human diseases and biological pathways. Nucleic Acids Res. 2014;42:D86-91.

28. Wang G, Guo X, Floros J. Differences in the translation efficiency and mRNA stability mediated by 59-UTR splice variants of human SP-A1 and SPA2 genes. Am J Physiol Lung Cell Mol Physiol. 2005;289:L497-508.

29. Andrés-Pons A, Rodríguez-Escudero I, Gil A, Blanco A, Vega A, Molina M, et al. In vivo functional analysis of the counterbalance of hyperactive phosphatidylinositol 3-kinase p110 catalytic oncoproteins by the tumor suppressor PTEN. Cancer Res. 2007;67:9731-9.

30. Maita N, Tsukimura T, Taniguchi T, Saito S, Ohno K, Taniguchi H, et al. Human a-L-iduronidase uses its own N-glycan as a substrate-binding and catalytic module. Proc Natl Acad Sci U S A. 2013;110:14628-33.

31. Bie H, Yin J, He X, Kermode AR, Goddard-Borger ED, Withers SG, et al. Insights into mucopolysaccharidosis I from the structure and action of a-L-iduronidase. Nat Chem Biol. 2013;9:739-45.

32. Bunge S, Kleijer WJ, Steglich C, Beck M, Schwinger E, Gal A. Mucopolysaccharidosis type I: identification of 13 novel mutations of the alpha-L-iduronidase gene. Hum Mutat. 1995;6(1):91-4.

33. den Dunnen JT, Dalgleish R, Maglott DR, Hart RK, Greenblatt MS, McGowanJordan J, et al. HGVS Recommendations for the Description of Sequence Variants: 2016 Update. Hum Mutat. 2016 Jun;37(6):564-9.

34. Chen X, Sullivan PF. Single nucleotide polymorphism genotyping: biochemistry, protocol, cost and throughput. Pharmacogenomics J. 2003;3:77-96.

35. Richards S, Aziz N, Bale S, Bick D, Das S, Gastier-Foster J, et al. Standards and guidelines for the interpretation of sequence variants: a joint consensus recommendation of the American College of Medical Genetics and Genomics and the Association for Molecular Pathology. Genet Med. 2015;17(5):405-24

\section{Submit your next manuscript to BioMed Central and we will help you at every step:}

- We accept pre-submission inquiries

- Our selector tool helps you to find the most relevant journal

- We provide round the clock customer support

- Convenient online submission

- Thorough peer review

- Inclusion in PubMed and all major indexing services

- Maximum visibility for your research

Submit your manuscript at www.biomedcentral.com/submit 